\title{
A Hidden Markov Model (HMM) Scheme for Lip based Identification Utilizing Vertical Grooves Angles
}

\author{
Alireza Shafii Mousavi \\ Islamic Azad University, Science and Research \\ Branch, Tehran, Iran
}

\author{
Houman Zarrabi \\ ICT Research Center \\ Tehran, Iran
}

\begin{abstract}
As the lips move too quickly, the shape of lips images may differ from one situation to another for an individual such as the cases of smiling or frowning. In this paper, we propose a novel approach to automatic classification of lips images which are captured by digital camera. We focus on the lower part of lips based on morphological features of the grooves. Image processing techniques are employed to replace the traditional lipprints to detect human identification with intelligent systems. Presented methodology takes lower part of the lips as an input to process their orientation of grooves. Morphological and structural features are considered to describe the lips. A Hidden Markov Model is proposed for classification and training. Results show the proposed algorithm is robust against scale changes, noises and multiple orientations of lip images for each person. With this approach $93.4 \%, 88.3 \%$ and $85.1 \%$ accuracy is achieved in classification of $5,10,15$ class respectively on 75 lip samples. Presented algorithm may contribute significantly for the development of applications related to human identifications or can be used as a supplement to lip identification systems (for example, methods which are using contour of the lips) to reduce the error rate of classification.
\end{abstract}

\section{Keywords}

Pattern recognition, Image processing

\section{INTRODUCTION}

The use of biometric features in identity recognition and verification has become a popular research area in the last decades since they can provide high level of security compared with the traditional access methods such as passwords and Personal Identity Numbers [1]. Lip print is an anatomical character of the human lips [2]. Identification of humans on the basis of lip wrinkles in lip prints is a type of forensic investigation known as cheiloscopy [3]. Lip prints are used in various applications such as identification of human in crime scenes, airport security or in smart phones for locking or unlocking or permission to access a certain applications using phone's camera. Suzuki and Tsuchihashi [4] reported two cases where lip prints have proven useful in identification of the criminal. They examined 1,364 subjects from 3 to 60 years old from both male and female and proved that lip characteristic are stable and unique for each examined person. Lip prints are important because of their uniqueness, except in monozygotic twins, and permanence makes it an important feature to human identification field [3], [5]. Research studies shows the uniqueness of lips print as they do not change throughout the life time [6], [7], [8], [9]. The importance of lip features as biometrics is reported in [1], too, where numerous lip-based features are evaluated. In this study, an approach based on the grooves of lips is presented.

\section{RELATED WORKS}

In [10] connected component analysis is used to analyze horizontal, vertical and diagonal groove features. Canny edge detection is used to enunciate all the groves present in the image. 20 samples of lip prints for 4 people are used and author reported 91 to 96 percent efficiency. The core of method in [11] is Hough transform to detect lines on the lips. As a result, a collection of line segments approximating the lip print pattern is obtained. Each segment is described by its length, angle and midpoint coordinates. 120 lip prints from 30 persons and 4 lip prints from each person is used to examine the effectiveness of method and $21,3 \%$ error rate is reported. In [12] lips shape and color features are considered in order to determine human identity. They used Zernike and $\mathrm{Hu}$ moments along with color features. Recognition rate of $86 \%$ for 114 lip prints from 38 persons is reported. A Hough transform based algorithm proposed in [13]. Sections were extracted using the Hough transform and an algorithm for searching for the sections lying on straight lines. The coefficient of similarity of average differences was used as the measure of the similarity between the sections. Error rate of $=$ $14,90 \%$ using 45 lip prints for 15 persons is reported. The approach presented in [14] uses the well-known DTW algorithm and Copeland vote counting method. Accuracy of $88.5 \%$ is reported on 120 lip prints for 4 lip prints sample for 30 persons. [15] proposed two methods. In Scale Invariant Feature Transform (SIFT) the features are extracted and matched with the features extracted from another lip image. If number of key points matched is above a threshold, the lip images are concluded to be from the same subject. In Speeded up Robust Features (SURF) the key points are detected using Hessian matrix. The features of one image to that of another image are matched by nearest neighbor method. Accuracy of 93\% and $94 \%$ for SIFT and SURF subsequently is reported by authors on 23 lip prints image from 10 people. Principal Component Analysis (PCA), and neural network classifiers is used for speaker identification by using lip features in [16]. PCA has been used for feature extraction; features are then used for training of the network by using different neural network classifiers such as Back Propagation (BP), Radial Basis Function (RBF) and Learning Vector Quantization (LVQ). A database of 12 subjects saying the first 4 digits in English is used and $91.07 \%$ accuracy in speaker recognition is obtained.

\section{PROPOSED ALGORITHM}

To date, most of the existing work concentrates either on lip movement, or the combination of lip movement and static features. There is little dedicated attention sufficiently paid into lower part of lip and its grooves alone or as a supplement to improve the rate of classification. In this study, we focus on the lower part of lips and morphological features of grooves are analyzed (see figure 1). The lines on the lip must be recognizable, not smudged; nether too dark nor too light and entire lower lip must be visible. Methods proposed in [13], [11] use Hough transform to detect curves on lips; in this study we use same technique to find vertical and diagonal lines (grooves) Where $\mathrm{H}$ and We are height and width of I and 
' = Hp / H. Hp is height of the pattern. Finally, I' (H', W) is desired image for further processing. Figure 2-d shows binary form of lip sample. Binarization process might cause disconnection of lines (grooves) so morphological operation is carried out to connect gaps between vertical or diagonal lines (see figure 2-e). The interest degree for diagonal lines in our algorithm is between 70 degrees and 110 degree based on horizontal X-axis. Vertical dilation of " times is performed for this purpose. Number of dilation must not be big as it might destroy the orientation of the grooves or lip and it must not be too small to become efficient. " is proportion of image height $(\mathrm{H})$ and practically " ' 0:1 H was outperformed. The result of dilation and erosion process based on defined " can be seen in figure 2-e.

Noise is a set of randomly distributed blackish specks that are mostly produced during binarization. For us in the human identification considering their lips, any feature other than the skeleton of the grooves is noise. Median filter is employed to remove salt and pepper noise (see figure 2-f), other unwanted components such horizontal lines will be removed or ignored in further steps of lower part of lips.

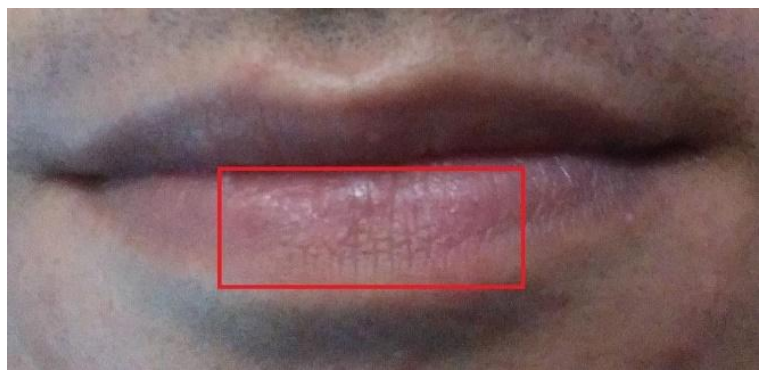

Fig. 1. Error rate in using codebook of six symbols in different number of segments

lip movement, or the combination of lip movement and static features. The proposed method includes a sequence of steps towards identification of human from lip image. First a series of pre-processing must be carried out to reduce unwanted noises and lip image normalization. The normalization procedure tends to standardize lip image by scaling, cropping and alignment of image. Segmentation process is performed to divide a lip image to a number of sub images in order to manage extraction of statistical and structural features in each zoon (segment). Extracted features are information that feeds to model for the purpose of training and classification. Hidden Markov model is employed as classifier. For each class one Discreet HMM is assigned and each model is trained using forward backward algorithm.

An internal database is generated for the purpose of training and testing. Images are captured by digital camera and lower part of lips are cropped manually using third party software in order to reduce the risk of border detection errors; it helps to achieve a pure classification rate regardless of possible lip detection error.

\subsection{Pre-processing}

lip images captured by digital camera are usually color images. Lip image is converted to gray level image and based on gray level histogram binarization process is carried out to discriminate foreground from background. Converting gray scale image into a binary form is called binarization. If the gray value of a pixel is more than the threshold value it is background, likewise it is foreground. The threshold is calculated by finding the dominant gray value in the scanned text which represents the background and then choosing the threshold value to be the midpoint or proportion of midpoint between the dominant value and the maximum gray value. Method described in [17] is used for binarization. Figure 2-b shows the gray level form of image in figure 2-a.

Cropped lower part lip images captured by digital camera might have different size and resolution, so scaling is performed to normalized the input image (I) to standard form $\left(I^{\prime}\right)$ using our mask pattern $\left(I_{p}\right)$ as follow

$H^{\prime}=\varphi * H . H^{\prime}=\varphi * W$

Where $H$ and $W$ We are height and width of $I$ and $\varphi=$ $H_{P} / H * H_{P}$ is height of the pattern. Finally, $I^{\prime}\left(H^{\prime} . W\right)$ is desired image for further processing. Figure 2-d shows binary form of lip sample. Binarization process might cause disconnection of lines (grooves) so morphological operation is carried out to connect gaps between vertical or diagonal lines (see figure 2-e). The interest degree for diagonal lines in our algorithm is between 70 degrees and 110 degree based on horizontal X-axis. Vertical dilation of " times is performed for this purpose. Number of dilation must not be big as it might destroy the orientation of the grooves or lip and it must not be too small to become efficient $\varepsilon$ is proportion of image height $(H)$ and practically $\varepsilon \approx 0.1 \mathrm{H}$ was outperformed. The result of dilation and erosion process based on defined $\varepsilon$ can be seen in figure 2-e.

Noise is a set of randomly distributed blackish specks that are mostly produced during binarization. For us in the human identification considering their lips, any feature other than the skeleton of the grooves is noise. Median filter is employed to remove salt and pepper noise (see figure 2-f), other unwanted components such horizontal lines will be removed or ignored in further steps.

Skeletonization algorithm is a parallel method which means that the new value obtained only depends on the previous iteration value. The purpose of the skeletonization (or thinning) is to reduce the number of pixels in the image and thus to speed up the processing in proposed method. The technique described in [18] is used for skeletonization of grooves in lips. Figure $2-\mathrm{g}$ shows the result of thinning process.

Pruning has a tough task of eliminating the hair-like noise, small chain codes, born to the skeleton of the grooves during the thinning process. The challenge is that the pruning should not touch the similar looking structures that are characteristic parts of the grooves. If foreground pixel be set to 1 and background be 0 then

$$
P_{n=} \sum_{x=-1}^{1} \sum_{y=-1}^{1}\left|p\left(x_{0}+\mid x \cdot y_{0}+y\right)\right| \mid \cdot n=1.2 \ldots . N
$$

Where $P$ is a set of points in the groove in lip and $P=$ $\left\{p_{1} \cdot p_{2} \cdot p_{3} \ldots . p_{n}\right\}$ and $x_{0}$ and $y_{0}$ are axis of each point, then the characteristic of each point in foreground considering their neighbor is as follow

$$
\left\{\begin{array}{cc}
\text { Junction } & \text { if }\left|P_{n}\right| \geq 4 \\
\text { Point }=\text { Head or Tail } & \text { if }\left|P_{n}\right| \geq 2 \\
\text { Point without junction } & \text { if }\left|P_{n}\right| \geq 3
\end{array}\right.
$$

Junction points is found based on the value of $p_{n}$. Removing small chain codes is performed by counting pixels in each chain code starting from junction point. As a result, no more than 2 chain code must be remained. It helps having pure grooves with fewer noises attached to them. At the last step of 
preprocessing a multiple dilation and erosion on thinned image is performed to reduce gaps in lines on the lip. Figure 2-h shows the result of the pruning and figure 2-h demonstrates the pruned image after smoothing using multiple dilations and re-thinning process. Figure 2-h is captured while the last dilation is performed.

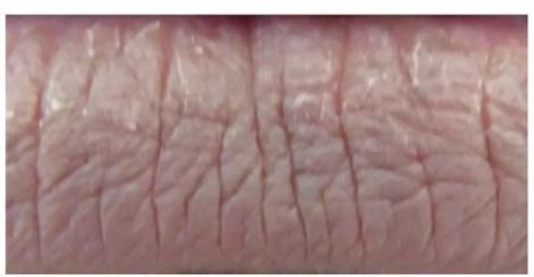

(a)

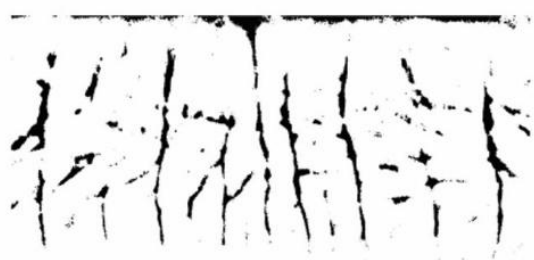

(d)

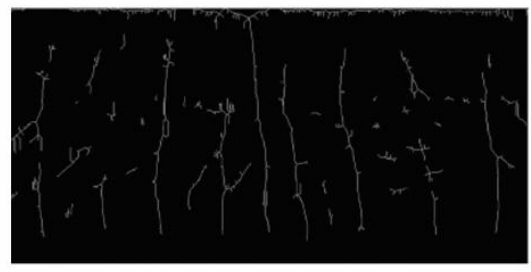

(g)

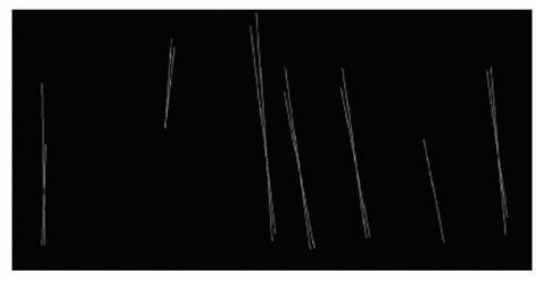

(j)

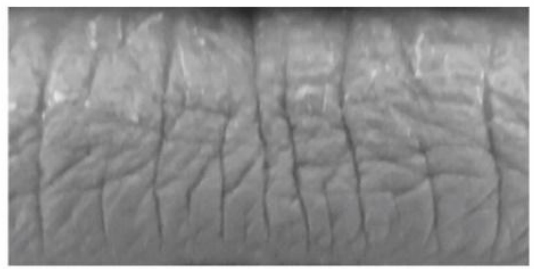

(b)

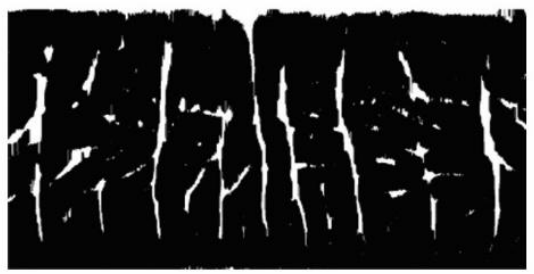

(e)

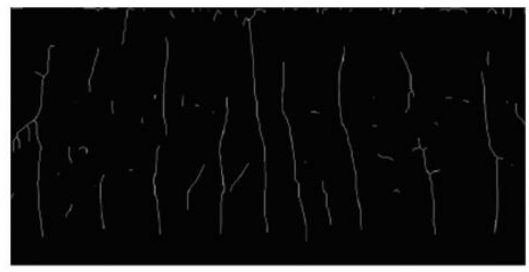

(h)

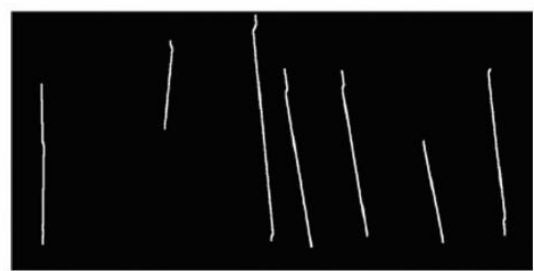

(k)

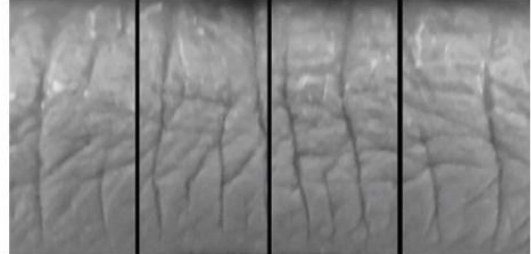

(c)

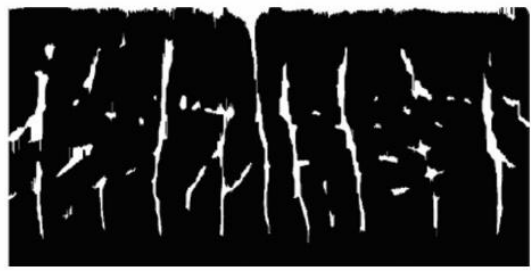

(f)

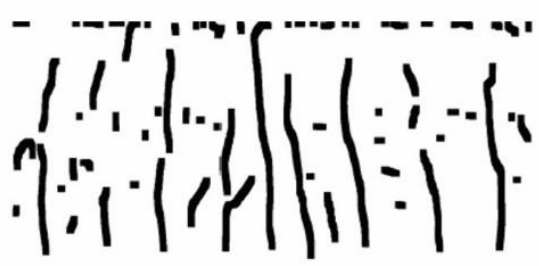

(i)

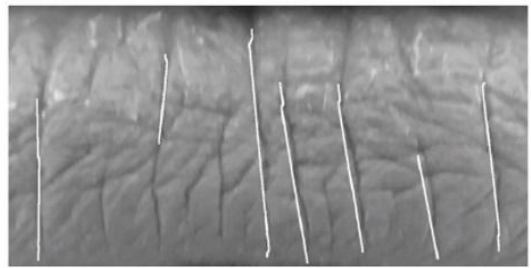

(I)

Fig. 2. Lip sample. (a): lower part of lip image (b): gray scale image (c): division of image into 4 segments (d): binarized form of lip image (e): lip image after applying morphological operations such as vertically closing operation (f): lip image after removing salt and pepper noises $(\mathrm{g})$ : Thinned image $(\mathrm{h})$ : Thinned image after pruning (i): last dilation operation and ready to employ Hough transform (j): result of Hough transform (k): smoothed form of lines (l): final result of Hough transform

\subsection{Segmentation and Feature Extraction}

The presented method for detection of lines on the lip images is based on Hough Transform. Hough Transform is a wellknown and documented method used in image processing. The main purpose of using this transform in this work is to detect grooves as a set of straight lines. Hough transform is not too sensitive to noise, variation and small gaps (disconnection) in a line and it is proved that this transform is very effective in many image processing applications such as finding skew angle in text line [19]. A survey and the details of Hough transform can be found in [20].
Lip image must be divided into a number of sub images. If Ns be the number of segments, so lip image can be represented as a sequence of segments as follow

$$
s=s_{1} \cdot s_{2} \ldots . s_{n}
$$

Segmentation is performed by dividing image vertically with equal width and height for all segments. $N_{s}$ should not be too length for line is $\Gamma$ which are defined as following

$$
\mu=\frac{2}{10} * H^{\prime} . \Gamma=\frac{1}{3} * H^{\prime}
$$

Where $H^{\prime}$ is height of the scaled image $I^{\prime}$. By considering 
axis of start and end point of each line using slope of the line with respect to horizontal $X_{a} x e s$, the incline degree of line can be calculated by

$$
\Theta=\frac{Y_{\text {second point }}-Y_{\text {first point }}}{Y_{\text {second point }}-Y_{\text {first point }}} * \frac{180}{\pi}
$$

As we are interested to process vertical like lines so we define an acceptable range for $\Theta$ as follow

$$
\alpha \leq \Theta \leq \beta
$$

Based on experimental results lines with the angles between 70 to 110 degree are valid line for further processing. Lines with the angle out of this range are ignored. Figure $2-\mathrm{j}$ shows the detected groves which are demonstrated as a line rather than curves. Smoothed form of figure 2-j is shown in figure 2$\mathrm{k}$.

Mean value of angles of lines for each segment must be calculated so for segment $n$ the mean value of angles $\left(M_{n}\right)$ is

$$
x=\frac{x_{1}^{n}+\cdots+x_{m}^{n}}{m}
$$

Where $m$ is number of lines in segment $n$ and $A_{m}^{n}$

Is the angle of line number $m$ in segment $n$. This system uses a code book to quantization of an angle feature. A pre-defined $N_{d}$ sets the number of symbols in the code book and calculates boundary as follow

$$
\lambda=\frac{|\beta-\alpha|}{N_{d}}
$$

Where $\lambda$ be denoted as boundary. Each symbols contains a range of angles as following algorithm

$$
\begin{aligned}
& \text { 1. Min }=\alpha \\
& \text { 2. For }\left(i=1 ; i<N_{d} ; i++\right) \\
& \text { 3. Min } \leq \text { Symboli< Min }+ \text { - } \\
& \text { 4. Min } \leftarrow \text { Min }+\lambda \\
& \text { 5. End }
\end{aligned}
$$

Codebook assigns a same symbol to lines with different angles within a same range. As a result, a sequence of observation $(O)$ must be generated by tracing segments from left to right for training and testing purpose. Additional features like number of lines in each segment will be discussed in experimental result section. Figure 2-1 shows final line detection and their accuracy on gray level form of original image.

\section{Table I. Age Distribution of Samples}

\begin{tabular}{lcccc} 
Gender & $18-21$ & $21-25$ & $25-29$ & $29-33$ \\
\hline Male & 2 & 2 & 3 & 2 \\
Female & 1 & 2 & 1 & 2 \\
\hline
\end{tabular}

Hidden Markov Model (HMM) is employed for classification purpose. The model gives a probability measure for a sequence of observations for a given state sequence. Details of HMM and its parameters can be found in [23], [24].

HMM model parameters are estimated in the training phase using the Baum-Welch algorithm (or known as Forward backward algorithm), followed by applying Viterbi decoding algorithm for scoring and calculating of the log-likelihood for each HMM. Class associated with the HMM of the highest log-likelihood is declared as the recognized class. Each HMM (each class) is trained separately. Number of states is same as number of segments and observations are symbols. Symbols are angles which are quantized by codebook.

Given an observation sequence $O$, our aim is to seek the class $L^{\prime}(L$ correspond to lips) that maximizes the a posteriori probability $P(L \mid O)$. Using the Bayes rule,

$$
\begin{aligned}
& L^{\prime}=\operatorname{argmax}_{l} P(L \mid O) \\
& =\operatorname{argmax}_{l} \frac{P(O \mid L) P(L)}{P(O)} \\
& =\operatorname{argmax}_{l} \frac{P\left(O \mid L^{\prime}\right) P\left(L^{\prime}\right)}{P(O)}
\end{aligned}
$$

$P(O \mid L)$ is the probability of $O$ (likelihood of the data) given class $L$. In the case of HMMs, $L$ is implicitly represented by its underlying model: $P(O \mid L)=P(O \mid \lambda) * P(O \mid \lambda)$ can be efficiently estimated in several ways depending on the task at hand. For this lip identification system which involve small number of classes, we build an HMM model $\lambda_{i}$ for each class and used the forward algorithm to estimate $\mathrm{P}\left(\mathrm{O} \mid \lambda_{i}\right)$ for $i=$

1..... $N_{L}$, where $N_{L}$ is the number of classes. In such a case, the people lip classes is said to be linear or flat.

\section{EXPERIMENTAL RESULT}

In order to create appropriate training sets, one must select a minimal set of samples which include various features of lip in each person. There are some publicly available dataset for face recognition such as [25], [26], However, after cropping lower part of lips it is observed that resolutions are very low and grooves are not visible.

In this study only lower part of lip is considered and samples were captured by digital camera. Lower parts of lips are manually extracted using Photoshop to decrease the detection error rate. This study is carried out on internal generated database consist of 15 person and 60 lip samples (see Table I) Since the main idea is relied on implementation of an intelligent system for Mobile phone to detect person identification for various purposes such as lock and unlocking the phone, image samples were taken from ordinary camera on the phone.

Since the main idea is relied on implementation of an intelligent system for Mobile phone to detect person identification for various purposes such as lock and unlocking the phone, image samples were taken from ordinary camera on the phone. The minimum resolution of lower part of lip images is $1260 * 650$ pixels. They are all visible without blurring and over lightening problem had been controlled as much as possible during photography. Lightening problem had been controlled as much as possible during photography.

In table II, shows that the comparison of proposed lip print recognition methods between different authors is described based on the outcome with different databases. 
Table II. Comparison of Proposed Lip Print Recognition Methods

\begin{tabular}{|c|c|c|}
\hline Authors and works & Results & Database \\
\hline Choras M. [12] & $\mathrm{EER}=14 \%$ & 114 lip prints ( 38 persons, 3 lip prints from each person) \\
\hline Porwik P., Orczyk T. [14] & $\mathrm{EER}=11,5 \%$ & 120 lip prints ( 30 persons, 4 lip prints from each person) \\
\hline Smacki L., Wrobel K., Porwik P. [11] & $\mathrm{EER}=21,2 \%$ & 120 lip prints ( 30 persons, 4 lip prints from each person) \\
\hline Wrobel K., Doroz R., Palys M. [13] & $\mathrm{EER}=14,90 \%$ & 45 lip prints (15 persons, 3 lip prints from each person) \\
\hline Sambit Bakshi, Rahul Raman, Pankaj K Sa [15] & $\begin{array}{l}\text { Accuracy }=93 \%(\text { SIFT }) \\
\text { Accuracy }=94 \%(\text { SURF })\end{array}$ & 23 lip prints from 10 person \\
\hline $\begin{array}{l}\text { Saptarshi Bhattacharjee, S Arunkumar, } \\
\text { Samir Kumar Bandyopadhyay [10] }\end{array}$ & Efficiency $=91-96 \%$ & 20 lip prints from 4 persons \\
\hline Proposed method & $\begin{array}{l}E E R=11.7 \% \text { for } 10 \text { person } \\
E E R=11.7 \% \text { for } 10 \text { person }\end{array}$ & 15 persons, 5 lip prints for each person \\
\hline
\end{tabular}

Table III. Experimental Result Considering different Interval Values, Boundaries and Segments on 10 Persons

\begin{tabular}{|c|c|c|c|c|c|c|}
\hline No of Intervals & $\begin{array}{c}\text { No of } \\
\text { Boundaries }\end{array}$ & Two Segments & Three Segments & Four Segments & Five Segments & Six Segments \\
\hline 10.00 & 4 & 7.3 & 68.0 & 63.1 & 59.7 & 57 \\
\hline 8.00 & 5 & 17.8 & 74.7 & 80.4 & 85.1 & 84.0 \\
\hline 6.666 & 6 & 23.8 & 77.2 & 88.3 & 82.3 & 73.2 \\
\hline 5.7143 & 7 & 11.1 & 78.6 & 82.5 & 79.1 \\
\hline 5.00 & 8 & 9.0 & 60.0 & 80.3 & 70.5 & 78.0 \\
\hline
\end{tabular}

It is necessary to practically examine the result with different number of segmentation with various boundary for angles $(\lambda)$ in order to create codebook. Some exceptions which were unpredictable after testing and classification are observed and based on preliminary experimental results it is observed that adding number of line as an additional feature in each segment to feature vector can improve the classification rate. Table III shows some experiments where codebook has 4 to 8 symbols. For each defined interval value different number of segmentation from two to six segments were examined.

Table III shows that dividing lip image into four segments using interval value of 6.666 outperforms. Figure 3 shows the error rate considering 5, 10 and 15 classes with codebook of six symbols (interval value of 6.666).

Based on figure 3, classifier produces less error rate for every number of classes $(5,10,15)$ by dividing lip image into four segments. Sensitivity to lightening is the main drawback of the proposed method. Since the algorithm works on grooves and extracts grooves line it is very depending on the visibility of grooves; on the other hand, the resolution of image must be high enough to detect details of the lines to obtain better classification rate. For large classes (for instance, lip images of people in a city), however, this approach is no longer feasible. The reason is threefold. First, considering one model for each class means that running the forward algorithm for each class separately could take a huge amount of time, which might not be affordable. Second, such a scheme may require a large amount of memory to store as much models as the size of the people and lip images.

For thousands of classes and thousands of lip images entries this can be a serious issue. Third, parameter estimation might get seriously degraded as a consequence of the lack of sufficient training lip image samples to adequately represent.

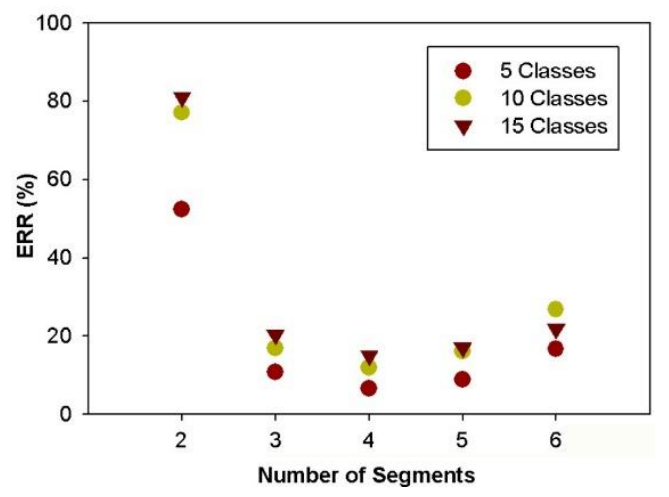

Fig. 3. Error rate in using codebook of six symbols in different number of segments

The author of this paper was keen to evaluate proposed method with other proposed algorithms in the same domain. It is not easy to compare the results obtained from the proposed method to the reported results where assumptions are different. On the other hand, there is no standard lip database available for researchers to compare the results. One method might have high rate of classification in one database but lower rate in other database. Since there is no standard dataset for groove detection no comparison can be made with other methods in line or grooves detection. However, as the final goal of lip-identification system is human identification, evaluation with previous works was made in table II. it seems that proposed method produces a competitive results. Interestingly, the result of this study is based on grooves in lower part of lips, not the entire lip; therefore, it is expected to produce higher accuracy considering the whole lip by extracting more statistical and topological features form shape of lips and involving upper part of lips as extra features. 


\section{CONCLUSION}

The proposed method is a new scheme towards lip identification based on angles of vertical and diagonal grooves. Grooves on the lip are represented as a set of lines with different angles. A series of preprocessing for normalization and removing noises is carried out and Hough transform is employed to detect grooves curve as straight lines. In our algorithm, angles of diagonal lines which are between 70 degrees and 110 degree based on horizontal Xaxis is our interest and lines that have angles out of this ranges are ignored. This study employs Hidden Markov Model kernel for classification. Baum-Welch algorithm is used to estimate the parameters of HMM. The lip images were taken from 15 individuals and 5 image of each person were collected to train and test the proposed model. One HMM is assigned to each class (person). With this approach 93.4\%, $88.3 \%$ and $85.1 \%$ accuracy was achieved in classification of 5, 10, 15 class respectively on 75 lip samples. Extraction of more features from border of lips and contributing upper part of lips will increase the classification rate, hence, decreasing error rate. Future work will be a hybrid approach of combination of this method considering lip border and involving upper part of lips and combination others algorithms to find better results.

\section{REFERENCES}

[1] S.-L. Wang and A. W.-C. Liew, "Physiological and behavioral lip biometrics: A comprehensive study of their discriminative power," Pattern Recognition, vol. 45, no. 9, pp. 3328 - 3335, 2012.

[2] H. F. E.-O. M. Wael M Saad, Assem H Kamel, "Genetic studies on the inheritance of lip prints in-cleft lip and palate,” Egypt J Plast Reconstr Surg, pp. 9-12, 2009.

[3] B. Karim and D. Gupta, "Cheiloscopy and blood groups: Aid in forensic identification," The Saudi Dental Journal, vol. 26, no. 4, pp. 176 - 180, 2014.

[4] T. Y. Suzuki K, "Two criminal cases of lip print,". ACTA Criminol, pp. Jpn 41: 61-64, 1975.

[5] Y. Tsuchihashi, "Studies on personal identification by means of lip prints," Forensic Science, vol. 3, no. 0, pp. $233-248,1974$.

[6] K. RK, "Lip prints an identification aid," Kathmandu Univ Med Je, vol. 38, no. 2, pp. 55-7, 2012.

[7] T. J. Augustine J, Barpande SR, "Cheiloscopy as an adjunct to forensic identification: A study of 600 individuals," J Forensic Odontostomatole, vol. 26, pp. 44-52, 2008.

[8] V. A. T. S. P. S. Bajracharya D, Mainali A, "Cheiloscopy: An aid in gender identification," J Nepal Dent Assoc, vol. 2, pp. 80-83, 2013.

[9] R. K. Saraswathi TR, Gauri Mishra, "Study of lip prints," Journal of Forensic Dent Science, pp. 28-31, 2009.

[10] S. Bhattacharjee, S. Arunkumar, and S. K. Bandyopadhyay, "Article: Personal identification from lip-print features using a statistical model," International Journal of Computer Applications, vol. 55, no. 13, pp. 30-34, October 2012.

[11] L. Smacki and K. Wrobel, "Lip print recognition based on mean differences similarity measure," in Computer Recognition Systems 4, ser. Advances in Intelligent and
Soft Computing. Springer Berlin Heidelberg, 2011, vol. 95, pp. 41-49.

[12] M. Chora, "The lip as a biometric," Pattern Analysis and Applications, vol. 13, no. 1, pp. 105-112, 2010.

[13] K. Wrobel, R. Doroz, and M. Palys, "A method of lip print recognition based on sections comparison," in Biometrics and Kansei Engineering (ICBAKE), 2013 International Conference on, July 2013, pp. 47-52.

[14] P. Porwik and T. Orczyk, "Dtw and voting-based lip print recognition system," in Computer Information Systems and Industrial Management, 2012, vol. 7564, pp. 191-202.

[15] S. Bakshi, R. Raman, and P. Sa, "Lip pattern recognition based on local feature extraction," in India Conference (INDICON), 2011 Annual IEEE, Dec 2011, pp. 1-4.

[16] A. Mehra, M. Kumawat, R. Ranjan, B. Pandey, S. Ranjan, A. Shukla, and R. Tiwari, "Expert system for speaker identification using lip features with pca," in Intelligent Systems and Applications (ISA), 2010 2nd International Workshop on, May 2010, pp. 1-4.

[17] W. Niblack, An Introduction to Digital Image Processing. Strandberg Publishing Company, 1985.

[18] S. L. L. Lam and C. Suen, "Thinning methodologies -a comprehensive survey," IEEE Transactions on Pattern Analysis and Machine Intelli-gence, vol. 14, pp. 869885, 1992.

[19] R. Shakoori, "A method for text-line segmentation for unconstrained arabic and persian handwritten text image," in Information Reuse and Integration (IRI), 2014 IEEE 15th International Conference on, Aug 2014, pp. $38-344$.

[20] J. Illingworth and J. Kittler, "A survey of the hough transform," Comput. Vision Graph. Image Process., vol. 44, no. 1, pp. 87-116, Aug. 1988.

[21] M. T. Parvez and S. A. Mahmoud, "Offline arabic handwritten text recognition: A survey," ACM Comput. Surv., vol. 45, no. 2, pp. 23:1-23:35, Mar. 2013.

[22] M. Khorsheed, "Off-line arabic character recognition - a review," Pattern Analysis and Applications, vol. 5, no. 2, pp. 31-45, 2002.

[23] L. Rabiner and B. Juang, "An introduction to hidden markov models," ASSP Magazine, IEEE, vol. 3, no. 1, pp. 4-16, Jan 1986

[24] L. Rabiner, "A tutorial on hidden markov models and selected applica-tions in speech recognition," Proceedings of the IEEE, vol. 77, no. 2, pp. 257-286, Feb 1989.

[25] A. S. Georghiades, P. N. Belhumeur, and D. J. Kriegman, "From few to many: Illumination cone models for face recognition under variable lighting and pose," IEEE Trans. Pattern Anal. Mach. Intell., vol. 23, no. 6, pp. 643-660, 2001.

[26] F. Solina, P. Peer, B. Batagelj, S. Juvan, and J. Kovac, "Color-based face detection in the "15 seconds of fame" art installation," in in Proceedings of Mirage 2003, (INRIA Rocquencourt, 2003, pp. 38-47. 